\title{
HACIA UNA TRAYECTORIA DE LA INSTITUCIONALIZACIÓN DE POLÍTICAS DE EQUIDAD DE GÉNERO EN ORGANISMOS PÚBLICOS CHILENOS
}

Carmen Andrade Lara 
CARMEN ANDRADE LARA

Socióloga, magíster en Gobierno y Política y especialista en el área de género y políticas públicas. Fue Ministra del Servicio Nacional de la Mujer (SERNAM). Actualmente se desempeña como Directora de Igualdad de Género de la Universidad de Chile. 


\section{HACIA UNA TRAYECTORIA DE LA \\ INSTITUCIONALIZACIÓN DE POLÍTICAS DE EQUIDAD DE GÉNERO EN ORGANISMOS PÚBLICOS CHILENOS}

\section{INTRODUCCIÓN}

Las agendas de género han ido avanzando paulatinamente en los diversos países de América Latina, enfrentando fuertes resistencias de los sectores que ven en ellas instrumentos que amenazan sus privilegios y las bases del orden de género. La construcción de sociedades e instituciones que garanticen igualdad de trato y de resultados a hombres y mujeres trasciende el campo de la acción pública, sin embargo, las políticas públicas pueden ser un buen instrumento para avanzar hacia esa dirección. De ahí deriva la importancia de diseñar, ejecutar y hacer sostenibles las denominadas políticas de igualdad entre hombres y mujeres.

Si bien existen evidentes diferencias entre los espacios gubernamentales y los universitarios, las experiencias y aprendizajes del proceso de institucionalización de la equidad de género en los Estados latinoamericanos -que revisaremos brevementepueden orientar el desarrollo de las iniciativas que buscan los mismos fines en el ámbito universitario y particularmente en la Universidad de Chile.

\section{EL PROCESO DE INSTITUCIONALIZACIÓN DE LA EQUIDAD DE GÉNERO EN EL ESTADO}

La institucionalidad de las políticas sociales, lo que también es aplicable a la institucionalidad de género, abarca el "conjunto de reglas de juego formales e informales (incluyendo las rutinas y costumbres organizacionales) que enmarcan el contenido y la dinámica administrativa y política de las políticas sociales”i (Repetto, Andrenacci, 2006). Su forma de organización, reglas y contenidos responden tanto a una racionalidad técnica, basada en diagnósticos, análisis de viabilidad técnicos y financieros, normas y reglamentos institucionales, como a una trama de decisiones políticas que dan cuenta de la importancia y visibilidad del "tema de género" en la sociedad y en las agendas públicas.

El desafío de institucionalizar la equidad de género ha estado presente en múltiples actores gubernamentales, movimientos feministas y de mujeres, y en instancias internacionales. A partir de las conclusiones de estos debates se estableció la necesidad y se dio curso a la creación de instituciones nacionales en 
diversos países de América Latina, denominadas Mecanismos para el Adelanto de las Mujeres (MAM), con potestades normativas, de formulación y coordinación de políticas orientadas a lograr la activa y plena participación social, política, económica y cultural de las mujeres.

Sus modalidades, presupuestos, jerarquías institucionales e inserción en las estructuras de gobierno fueron heterogéneas. Se organizaron ministerios, servicios nacionales, secretarías y fundaciones que dependían directamente del Ejecutivo o de ministerios sectoriales, generalmente del área social. Estos factores fueron gravitantes en su capacidad de incidir en el conjunto del accionar público, comprobándose que lograban mayor influencia si se ubicaban en los niveles superiores de la toma de decisiones, contaban con suficiente autonomía política, despliegue territorial en unidades descentralizadas, presupuesto y personal suficiente para operar, y marcos normativos que aportaban a su sostenibilidad.

Considerando sus diferencias y limitaciones, la evaluación de sus resultados mostró que estos mecanismos institucionales contribuyeron a "reducir las desigualdades, mejorando la condición de las mujeres, a través de reformas legales, diseño e implementación de programas específicos, producción de conocimientos y políticas públicas con enfoque de género; (y que) cumplen un rol fundamental en la incorporación del enfoque de género en las políticas públicas" (CIM, OEA, 2008) . Sin embargo, a diferencia de otros organismos estatales, su estabilidad ha dependido en general de factores externos y coyunturales, tales como cambios de gobierno, redefinición de orientaciones gubernamentales y cambios en las correlaciones de fuerzas culturales y políticas.

La especificidad de las políticas públicas impulsadas por los MAM consiste en enfrentar la desigualdad de hecho y de derecho entre mujeres y hombres, así como sus distintas manifestaciones, como problema de intervención. En sus diseños se parte de la base de que el Estado y sus instituciones no son neutrales en la configuración de las relaciones sociales de género sino que, incluso sin proponérselo, pueden reafirmar o incrementar las desigualdades o bien contribuir a reducirlas y erradicarlas.

Durante décadas y promovidos por organismos internacionales, en diversos países de América Latina se ejecutaron los llamados planes y programas para mujeres con el objetivo de integrarlas al desarrollo, potenciando su papel productivo. Sus evaluaciones demostraron que si bien estos daban respuestas a necesidades inmediatas y concretas, como la pobreza, la violencia, la falta de acceso a la educación y a la salud materna, no modificaban la subordinación de las mujeres ni las asimetrías de poder que caracterizan los sistemas patriarcales. Por ello se pusieron en práctica nuevas políticas de igualdad de oportunidades, de acción positiva y de paridad, que ampliaron el ejercicio de derechos legales y favorecieron el acceso de las mujeres al empleo, la educación y la representación política, entre 
otros. Sin embargo, tuvieron menores efectos en transformar la organización social que sustenta la discriminación.

Por lo tanto, y tomando en cuenta que todas las relaciones sociales entre hombres y mujeres y su participación social, política y económica está condicionada por el sistema de género, se evidenció que el conjunto de políticas públicas, desde las macroeconómicas hasta las locales, tienen impactos diferenciados en la vida de ambosiii (Astelarra, 2004). Esto hizo que cobrara fuerza la estrategia de empoderamiento de las mujeres y de transversalización "para hacer de las preocupaciones y experiencias, tanto de mujeres como de varones, una dimensión integral del diseño, implementación, monitoreo y evaluación de políticas y programas en todas las esferas, política, económica y social, de modo que ambos géneros se beneficien igualitariamente" (ECOSOC, 2002) ).

En este camino, los movimientos de mujeres y feministas jugaron un rol decisivo dado que visibilizaron las desigualdades y discriminaciones e interpelaron a los Estados a través de movilizaciones sociales e interlocución política, con el objetivo de que las incorporaran en la agenda pública. Su participación en instancias de diálogo representativas de la diversidad de organizaciones y movimientos ha sido también relevante para "mejorar la articulación entre actores y la concertación de políticas públicas de género (...) los cursos de acción pública involucran y comprometen no solo a los organismos estatales sino también a la sociedad, asegurando legitimidad, pluralismo y pertinencia en las intervenciones"v (INSTRAW, 2010).

Las conferencias y convenciones internacionales de los años ' $80-' 90$ favorecieron en cierta medida la institucionalización de la equidad de género porque sus conclusiones, vinculantes para las legislaciones y políticas nacionales, comprometieron a los Estados que las suscribieron. Entre estas destacan la Convención sobre la Eliminación de Todas las Formas de Discriminación Contra la Mujer (CEDAW) y la Convención Interamericana para Prevenir, Sancionar y Erradicar la Violencia Contra la Mujer de Belem do Pará.

La institucionalización también ha estado condicionada por las voluntades políticas de las autoridades públicas y por las concepciones y relatos culturales hegemónicos que se instalan y circulan en el Estado. Por ello ha encontrado condiciones favorables para desarrollarse precisamente en contextos nacionales de reconstrucción de la democracia, después de décadas de dictaduras militares, de pacificación luego de largos conflictos armados y de globalización que difundió nuevos modelos culturales. El caso chileno así lo reafirma; la etapa postdictadura de democratización y recuperación de derechos ciudadanos constituyó una ventana política que el movimiento de mujeres supo utilizar para incorporar muchas de sus reivindicaciones en la agenda de gobierno, entre ellas, la creación de institucionalidad de género. 
Otros factores gravitantes fueron los procesos de modernización y de fortalecimiento del rol de los Estados nacionales, fuertemente debilitados por las políticas de ajuste estructural llevadas a cabo durante la llamada "década perdida". "La singularidad de las agendas de género en la región radicó en haberse anticipado a la crítica al neoliberalismo, demandando un rol activo del Estado que estaba en retirada"vi (Guzmán, Montaño, 2012).

El carácter multidimensional de las desigualdades y discriminaciones planteó nuevos desafíos a la gestión pública debido a que las políticas de igualdad requieren de formas de coordinación intersectorial y transversal, lo que suele contradecir las lógicas administrativas compartimentadas del Estado. Por ello, con frecuencia se percibe que sus modelos de gestión resultan difíciles de replicar y se opta por implementar sólo políticas de corto alcance o planes piloto que no logran trascender a todo el país.

La necesaria intersectorialidad, la participación ciudadana en su ejecución y sobre todo su capacidad de transformación sociocultural son elementos constitutivos de las políticas mencionadas. Estos rasgos las conducen generalmente a estar "bajo sospecha" y sometidas al escrutinio, principalmente de los denominados "grupos de veto" que actúan en defensa del statu quo. Un ejemplo claro de ello ha sido la difícil instalación y las fuertes resistencias a los planes y legislaciones nacionales que garanticen el pleno ejercicio de los derechos sexuales y reproductivos. Por estas razones enfrentan una doble tensión al compararlas con otras políticas públicas; deben demostrar su eficacia cumpliendo cabalmente los objetivos para los cuales fueron creadas y a la vez sustentarse en una institucionalidad que las legitime y proyecte. Si uno de estos aspectos falla, el riesgo de su discontinuidad es inminente. Paradojalmente, la institucionalización de estas iniciativas les confiere estabilidad y al mismo tiempo abre caminos para modificar las culturas y estructuras institucionales en las que se instalan.

Los mecanismos institucionales creados y los movimientos sociales han contribuido a incorporar nuevas concepciones y formas de trabajo, presionando a los Estados a intervenir más activamente en el campo de las desigualdades. "(Este proceso) enlaza dimensiones discursivas sobre el orden de género con la construcción de sistemas interactivos con otros actores dentro y fuera del Estado" (Benavente, Valdés, 2014) vii.

Las experiencias nacionales han seguido cursos distintos y muestran que la creación de organismos institucionales especializados, la puesta en marcha de la estrategia de transversalización y la participación activa de los actores sociales han sido cruciales para la institucionalización de la equidad de género en el Estado, ya que esta constituye la materialización de relaciones políticas, prácticas sociales y visiones del mundo que se legitiman como cosas públicas por medio de iniciativas precedidas por luchas políticas (Guzmán, 2006) ) $^{\text {viii }}$. 
Con todas sus complejidades, estos procesos se han ido desarrollando con mayor o menor profundidad, con avances y retrocesos, y seguirán vigentes en la medida en que también lo hagan las desigualdades sociales que intentan enfrentar, así como los movimientos de mujeres y feministas y los mecanismos institucionales nacionales que los impulsan y sostienen.

\section{EL PROCESO DE INSTITUCIONALIZACIÓN DE LAS POLÍTICAS DE GÉNERO EN LA UNIVERSIDAD DE CHILE: AVANCES Y DESAFÍOS}

"La Universidad de Chile ha sido pionera en abordar las inequidades de género. Desde finales del siglo XIX con Eloísa Díaz, estudiante de medicina que debía asistir a sus clases tras un biombo y luego, a mediados del siglo XX con las primeras catedráticas como Amanda Labarca, Elena Caffarena (...) hasta los actuales Centros de Estudios de Género, nuestra universidad ha sido vanguardista en la incorporación de las mujeres y, asimismo, en recoger sus problemas y demandas" ix (Universidad de Chile, 2013).

Sin embargo, la inclusión de la perspectiva de género al interior de la propia Universidad está aún pendiente, lo mismo que en las diversas instituciones de educación superior, que expresan y reproducen las mismas contradicciones presentes en las sociedades en las que se insertan. "A pesar de que la mayor parte de los avances en temas de género ha salido de la producción académica de las universidades, no han sido estas instituciones las que han acogido la temática y sus procesos de institucionalización al interior de sus comunidades”x (Buquet, 2011).

Asumir este desafío significa incorporar nuevas concepciones en la gestión de la Universidad y establecer la igualdad entre hombres y mujeres y el fin de las discriminaciones como propósito institucional que se integra en las políticas, estrategias, programas, actividades administrativas y financieras e incluso en la cultura institucional. No cabe duda de que se ha avanzado, pero aún falta un largo camino para alcanzar estos propósitos.

Tomando como referencia a Székely $(2006)^{x i}$, que caracteriza las políticas institucionalizadas en función del cumplimiento de determinados requisitos políticos, técnicos y normativos, se podría considerar que las políticas de equidad de género, en términos operacionales, estarían institucionalizadas si en el ámbito universitario existiera:

- un acuerdo institucional para abordar las discriminaciones y brechas de género como objeto de intervención de la gestión universitaria,

- definiciones y conceptualizaciones de los problemas de género enmarcados en valores y principios universitarios, 
- mecanismos específicos encargados de impulsar la equidad de género y un marco normativo, legal y reglamentario que los sustente,

- planes, objetivos y metas de igualdad de género, así como instrumentos e indicadores para evaluarlas,

- presupuesto estable, proporcional a las funciones asignadas, e instancias de participación de los diversos actores universitarios.

Un paso importante se produjo cuando en 2012 la autoridad universitaria tomó la decisión de conformar la Comisión de Igualdad de Oportunidades de Género, coordinada por académicas feministas y destinada a "analizar el funcionamiento de la Universidad desde la perspectiva de la equidad de género, con el fin de proponer medidas que permitan avanzar en la profundización del compromiso en favor de la igualdad que la Universidad de Chile ha adquirido"xii (Universidad de Chile, 2013).

Esta comisión, junto con levantar un diagnóstico preliminar acerca de la participación de hombres y mujeres en la Universidad, propuso a las autoridades la creación de un organismo institucional para profundizar el conocimiento sobre la materia y promover políticas antidiscriminatorias, es decir, abrió el camino para problematizar y transformar la construcción cultural universitaria. "Plantear la necesidad de mirar a la propia institución y a los sujetos genéricos que la conforman (...) requiere tomar una posición política que redunde en medidas para transformar la desigualdad estructural al interior de la institución"xiii (Cerva, 2017).

Así se generó, a fines de 2013, un pequeño mecanismo, la Oficina de Igualdad de Oportunidades de Género, dependiente de la Vicerrectoría de Extensión y Comunicaciones, en tanto que la Comisión de Igualdad de Oportunidades asumió el carácter de asesora. Uno de sus primeros pasos fue la realización de un estudio denominado Del biombo a la cátedra: igualdad de oportunidades de género en la Universidad de Chile, cuyos resultados reafirmaron la existencia de relaciones sociales marcadas por notorias asimetrías y brechas: i) segregación horizontal expresada en menor proporción de mujeres en el estamento académico, "masculinización” de las carreras científicas y tecnológicas, y "feminización" de las carreras sociales; ii) segmentación vertical manifestada en subrepresentación femenina en los niveles de decisión y en las jerarquías académicas superiores; iii) brechas salariales por sexo en ambos estamentos en desmedro de las mujeres; iv) barreras de género en el desarrollo de la carrera académica, estudiantil y funcionaria; y v) violencia de género y acoso sexual que afectan mayoritariamente a las estudiantes y que escasamente se denuncian.

A partir del estudio mencionado se ejecutaron múltiples actividades para socializar sus contenidos tanto en las facultades e institutos como en el acto central de conmemoración del 8 de marzo (Día Internacional de la Mujer) de 2014. En los diálogos hubo reacciones tales como: “eestán seguras de los datos?”, "esto ocurre 
en la Universidad, pero no en mi facultad", "las diferencias de salarios no tienen nada que ver con variables de género sino con productividad académica”. A pesar de ellas, contar con información empírica posibilitó visibilizar y debatir situaciones que sin ser desconocidas, habitualmente no se problematizaban, y cuestionar la idea generalizada de que las universidades se rigen por un "modelo de funcionamiento supuestamente igualitario (ignorando que) el contexto patriarcal también se manifiesta en los espacios en donde el conocimiento se produce y éste también está determinado por estructuras y dinámicas organizacionales que refuerzan una cultura organizacional que reproduce estereotipos y patrones sexistas" ${ }^{\text {xiv }}$ (Cerva, 2017),

Considerando que la identificación y conceptualización de las desigualdades entre hombres y mujeres presentes en la Universidad no aseguraban la decisión de abordarlas, se consideró necesario implementar diversas estrategias para que se reconocieran como asuntos que involucraban a la comunidad y que era necesario incluir en la agenda universitaria.

Una de estas estrategias fue la conformación de instancias estables de participación triestamental, que se sumaron a la Comisión de Igualdad de Oportunidades de Género ya existente:

\section{I. la Comisión de Prevención del Acoso Sexual,}

II. la Comisión de Corresponsabilidad Social en el Cuidado, y

III. diversos grupos de trabajo abocados a proponer cambios normativos, modelos de atención de víctimas de acoso sexual y planes de comunicación y de capacitación de funcionarios/as.

Estos colectivos conformadas por representantes de la Federación de Estudiantes, la Federación de Funcionarios/as, las Secretarías de Sexualidades y Género (SESEGEN), el Senado Universitario, los centros de estudios de género, el Movimiento de Madres y Padres Universitarios (MAPAU) y diversas direcciones universitarias y académicas feministas reforzaron y ampliaron las acciones de la Oficina de Igualdad de Oportunidades de Género, permitieron incluir las necesidades e intereses de las estudiantes, académicas y funcionarias en las medidas y planes y apoyaron y legitimaron las propuestas que se desarrollaron.

Con el propósito de reforzar académicamente a la Oficina de Igualdad, la Vicerrectoría de Extensión y Comunicaciones creó la Cátedra Amanda Labarca, destinada a realizar docencia, investigación y extensión. El trabajo liderado por esta cátedra, con colaboración de la Oficina y de los centros de estudios de género, se tradujo en cursos de formación general, diversos seminarios y actividades de extensión. 
Las/os integrantes de la Oficina y de la Comisión que la asesora debatieron ampliamente para dilucidar si era más adecuado elaborar una propuesta global de política universitaria de igualdad o bien partir por iniciativas que respondieran a problemas específicos relevados por distintas organizaciones tales como el MAPAU, las SESEGEN y las asociaciones gremiales. Se optó por la segunda vía, estimando que el proceso para construir la política era tanto o más importante que el producto final. Por tanto, se consideró que el diseño de planes posibles de ejecutar en plazos razonables abriría el debate en la comunidad acerca de los derechos de las mujeres y las barreras para ejercerlos y contribuiría a generar un clima favorable para proyectar una política de igualdad elaborada participativamente y que hiciera sentido a la mayoría de la comunidad universitaria.

Se formularon entonces políticas universitarias para enfrentar i) la violencia y el acoso sexual y ii) las inequidades entre hombres y mujeres en el desarrollo de la carrera. En paralelo se ejecutó una amplia gama de actividades de información, sensibilización y debate, tales como talleres, coloquios, seminarios, edición de documentos, etc., para difundirlas y afirmar su implementación en todos los campus. El conjunto de acciones desplegadas fue sumando aliados/as, abriendo espacios de legitimación de la equidad de género y del mecanismo institucional creado para impulsarla.

En primer lugar, en conjunto con la respectiva comisión triestamental, se elaboró la Política de Prevención del Acoso Sexual, un fenómeno recurrente en la Universidad de Chile al igual que en el contexto universitario, tal como lo demuestra un estudio de la Oficina de Igualdad de Oportunidades de Género'. La política de prevención reconoce esta conducta como una manifestación de la violencia ejercida contra las mujeres arraigada en la cultura patriarcal, que afecta su integridad personal y limita el ejercicio de sus derechos. La iniciativa incluye:

I. acciones de sensibilización e información,

II. cursos de formación en género y violencia,

III. una Oficina de Atención de Víctimas que entrega asesoría y acompañamiento jurídico-social,

IV. un servicio de atención psicológica especializada,

1. $26 \%$ de las personas encuestadas han conocido historias de acoso en espacios universitarios o actividades relacionadas y 14,7\% las han sufrido directamente en su trayectoria universitaria. Las afectadas son mayoritariamente mujeres estudiantes que son acosadas por sus profesores o por sus compañeros de estudios. 
V. la realización de modificaciones reglamentarias y normativas para tipificar y sancionar el acoso y la violencia sexual, incluyendo la generación de un Protocolo de Actuación,

VI. cursos de capacitación de fiscales y actuarios para que investiguen estas conductas, y

VII. sistematización de información sobre denuncias y procedimientos de investigación y realización de estudios sobre el tema.

En segundo lugar, con la participación de la Comisión de Corresponsabilidad Social se elaboró una política universitaria orientada a la conciliación entre vida laboral, familiar y personal desde una perspectiva de corresponsabilidad social en el cuidado de niños/as. Dicha política reconoce que esta labor genera valor para toda la sociedad, pero culturalmente se atribuye como responsabilidad casi exclusiva de las mujeres. Y aunque ellas se han incorporado masivamente al mundo público, no se han redistribuido los roles familiares y domésticos entre hombres y mujeres, ni se ha producido una respuesta pública pertinente a las actuales demandas de cuidado.

Las académicas y funcionarias de la Universidad de Chile también enfrentan estas dificultades debido a que las altas exigencias académicas y laborales se suman a las responsabilidades familiares, lo que a diferencia de sus pares varones, limita su desarrollo profesional y retarda los procesos para avanzar en su formación y en las distintas jerarquías universitarias. Por su parte, las estudiantes que son madres no cuentan con facilidades académicas o bien estas dependen de cada profesor; la oferta pública de cuidado infantil se focaliza prioritariamente en sectores sociales de mayor vulnerabilidad en tanto que la oferta privada es de alto costo. Todo ello redunda en la reducción de sus cargas académicas, postergación de sus estudios y excesivos esfuerzos personales.

\section{La Política de Corresponsabilidad contempla:}

I. la instalación de cuatro salas cunas y jardines infantiles en campus universitarios con acceso preferente de hijos/as de funcionarias y estudiantes, mediante un convenio con la Junta Nacional de Jardines Infantiles (JUNJI),

II. un nuevo reglamento estudiantil que garantiza derechos de pre y postnatal, permisos por enfermedad de hijos/as, flexibilidad académica y prioridad en la toma de ramos para estudiantes madres/padres, entre otras medidas, y finalmente,

III. un conjunto de modificaciones a los reglamentos que regulan la carrera y la evaluación académica para corregir los aspectos inequitativos. 
Las propuestas de políticas debieron ser presentadas en diversas instancias directivas, lo que permitió visibilizar y analizar las inequidades de género en lugares institucionales donde esto no era habitual. Finalmente, y luego de un largo proceso, estas fueron aprobadas unánimemente por el Senado Universitario y respaldadas por el Consejo Universitario ${ }^{2}$. "Es fundamental generar espacios de debate al interior de la institucionalidad pública. Incluir el género no es sólo incluir una norma, un procedimiento, es tratar de empezar a re-pensar las maneras de concebir la realidad y actuar sobre los problemas, para modificarlos" (Guzmán 2006) ${ }^{\mathrm{xv}}$.

En estas presentaciones, respaldadas por abundante información, evidencia empírica y experiencia comparada, se generaron debates y se fueron expresando opiniones favorables, lo que no es extraño en un ambiente cultural como el de la Universidad de Chile. En este clima resultaron clave las expresiones del Rector Ennio Vivaldi, quien marcó la voluntad política institucional: "Cuando se combate cualquier tipo de discriminación, se está defendiendo no sólo a los discriminados, sino a toda la sociedad, a la humanidad si se quiere. Este es un tema que une a todas las personas que quisieran ver un mundo más justo, un mundo de progreso basado en valores. Por eso todos debemos agradecer el valor de cada mujer y por ende sus derechos" 3 .

La implementación de estas políticas no ha estado exenta de dificultades. Ha habido serias deficiencias en su difusión y la utilización de distintos canales de información no ha sido suficiente para informar a toda la comunidad sobre los derechos y beneficios que contemplan y sobre las concepciones en las que se fundan. Esto ha sido particularmente crítico en el caso del Protocolo de Actuación ante Casos de Acoso Sexual, Laboral y Discriminación Arbitraria. Por esta razón se ha conformado un grupo de trabajo triestamental para diseñar y poner en marcha un plan y una estrategia comunicacional vinculada principalmente con la prevención de la violencia de género.

La Universidad cuenta con todas las condiciones y sistemas que le permiten cumplir con estándares de excelencia sus funciones de docencia, extensión e investigación. Sin embargo, estas nuevas propuestas no forman parte de las "rutinas institucionales" e implican tareas, funciones y formas de coordinación para las cuales no siempre existen los procedimientos y capacidades institucionales.

Por ejemplo, la Política de Corresponsabilidad Social requiere hacer seguimiento a la construcción de jardines infantiles, establecer comodatos para ceder terrenos,

2. Cuerpo directivo presidido por el Rector e integrado por los/as vicerrectores/as, decanos/as, directores/as de institutos y Director Jurídico.

3. Preámbulo del Rector Ennio Vivaldi en documento Política para Prevenir el Acoso Sexual en la Universidad de Chile. Santiago, 2016. 
coordinar con JUNJI el ingreso de niños/as a los establecimientos, instalar lactarios, hacer catastros de madres/padres universitarios, implementar un inédito permiso postnatal a estudiantes, etc. Es decir, un conjunto de actividades no habituales y que además suponen coordinación fluida entre distintas unidades cuya gestión ha sido ajena a políticas de género: los decanatos, la Dirección de Infraestructura, la Dirección de Bienestar, la Dirección Jurídica, entre otros, que se insertan en un contexto conocido en la Universidad como un "archipiélago de facultades e institutos".

Existen también limitaciones que derivan de las insuficiencias y falta de pertinencia de las políticas públicas para el contexto universitario. Por ejemplo:

- el acoso y la violencia sexual en espacios educativos -entre compañeros o entre profesores y estudiantes- no han sido considerados en las leyes de violencia que sólo regulan la violencia intrafamiliar o el acoso sexual en espacios laborales,

- los sumarios administrativos que se aplican en las instituciones públicas como la Universidad de Chile resultan totalmente inadecuados para investigar y sancionar la violencia de género y discriminación arbitraria. No se tipifica el acoso sexual entre profesores y alumnas, sólo se reconocen derechos a los inculpados y no a las denunciantes, entre otras.

- existen pocos programas especializados en la atención de víctimas de acoso sexual, por lo que hay que considerar largos tiempos de espera,

- los escasos programas públicos de intervención educativa con hombres agresores sólo consideran a adolescentes o a adultos que ejercen violencia en contexto intrafamiliar, lo que deja fuera a estudiantes universitarios que cometen acoso,

- las salas cunas de JUNJI (0-2 años) que se construyen en los campus universitarios no pueden ser utilizadas por hijos/as de funcionarias y académicas porque la ley laboral no lo permite; ellos/as sí pueden acceder a los jardines infantiles (2-4 años). Se produce entonces el absurdo de que contando con establecimientos públicos, gratuitos y de calidad, la Universidad debe pagar la atención de los/ as hijos/as de su personal en salas cunas privadas.

El carácter innovador de estas propuestas las ha enfrentado también a restricciones, ausencias e insuficiencias normativas, lo que ha obligado a seguir un largo proceso de revisión y modificación de normas y reglamentos. Para ejecutar la Política de Corresponsabilidad Social en el Cuidado hubo que incorporar en el reglamento estudiantil este tipo particular de prestaciones que no estaban consideradas; para implementar la Política de Prevención del Acoso Sexual fue necesario hacer cambios al reglamento de estudiantes, ya que no incluía estas 
prácticas entre las conductas sancionables. Se debió elaborar un Protocolo de Actuación ante casos de acoso sexual, acoso laboral y discriminación arbitraria, y se dictaron diversos decretos para crear nuevos órganos institucionales que aseguraran la implementación de las medidas. Asimismo, se formuló una circular desde Rectoría para garantizar el reconocimiento del nombre social de los estudiantes trans en todas las instancias de la Universidad, lo que demandará la revisión de los sistemas de información. Actualmente, los cambios normativos continúan: se encuentra en revisión el Reglamento de Jurisdicción Disciplinaria de Estudiantes y se está reformulando el protocolo de actuación para adaptarlo a la nueva ley de universidades estatales.

La falta de pertinencia de los reglamentos y normas se expresa también en materias de género. Por ejemplo, el reglamento de estudiantes consideraba entre las conductas sancionables el plagio o la falta de respeto a los emblemas universitarios y nunca se planteó el reproche al acoso o la violencia sexual que afecta el ejercicio de derechos fundamentales, particularmente de las mujeres.

Continuar con nuevos planes y medidas de equidad supondrá perseverar en las modificaciones normativas, de los reglamentos de evaluación y jerarquización académica, de modo de igualar las oportunidades entre hombres y mujeres en el desarrollo de la carrera, de los reglamentos de remuneraciones para enfrentar las brechas salariales que afectan a académicas y funcionarias ${ }^{4}$, de los sistemas de elección de autoridades para aumentar la participación femenina en los cuerpos directivos, entre muchas otras.

Frente a las limitaciones legales se realizaron múltiples acciones de incidencia legislativa: se realizó una presentación en el Parlamento y se logró incluir indicaciones en la Ley de Universidades Estatales para mejorar los procesos de investigación, reconocer el acoso sexual entre profesores y alumnas y equilibrar los derechos de los acusados y las denunciantes ${ }^{5}$. También se realizaron coordinaciones con el

4. El Senado Universitario ha presentado un proyecto de nuevo reglamento de remuneraciones que aborda principalmente las desigualdades salariales entre unidades académicas pero no se hace cargo expresamente de las brechas salariales por sexo.

5. "Con todo, para el personal académico y no académico de las Instituciones de Educación Superior del Estado, las prohibiciones establecidas en el Estatuto Administrativo relativas a actos atentatorios a la dignidad de los demás funcionarios, incluido el acoso sexual, el acoso laboral y la discriminación arbitraria, se entenderán también referidas a conductas del mismo tipo en contra de estudiantes, servidores a honorarios y de toda persona vinculada, de cualquier forma, a las actividades de la institución. Además, en los procedimientos instruidos para determinar la responsabilidad administrativa en este tipo de casos, las víctimas y personas afectadas por las eventuales infracciones tendrán derecho a aportar antecedentes a la investigación, a conocer su contenido desde la formulación de cargos, a ser notificadas e interponer recursos en contra de los actos administrativos en los mismos términos que el funcionario inculpado". Art. 34, Ley 21094 sobre universidades estatales. 
Ministerio de la Mujer y la Equidad de Género para incluir la especificidad del acoso sexual en contexto educativo en el proyecto de ley de violencia integral que se encuentra en el Parlamento 6 .

Asimismo, en el marco de la discusión parlamentaria del proyecto de ley que modifica el Código del Trabajo en materia del derecho a sala cuna, se formularon propuestas, vía indicaciones parlamentarias, para que las funcionarias del Estado pudieran optar entre hacer uso de las modalidades que ofrece la actual ley laboral o de las salas cunas de JUNJI. Estas fueron aprobadas en la Cámara de Diputados pero aún se encuentra pendiente su trámite en el Senado ${ }^{7}$.

El conjunto de acciones desarrolladas fue incrementando la sensibilización, la información y los apoyos a las políticas de género e hizo patente la necesidad de ampliar las intervenciones y profundizar la agenda de género universitaria. Estos desafíos dejaron en claro que la Oficina de Igualdad de Oportunidades no era suficiente para responder a la magnitud de las tareas que debían emprenderse.

En consecuencia, a principios de 2018 se creó la Dirección de Igualdad de Género (DIGEN) y la Oficina de Atención para Personas Afectadas por Acoso Sexual, Violencia de Género y Discriminación Arbitraria. La DIGEN depende directamente de la Rectoría y cuenta con un Consejo Consultivo integrado por los vicerrectores y las vicerrectoras.

Sus funciones, explicitadas en su decreto de creación, consisten en elaborar y monitorear el cumplimiento de políticas, medidas y normativas destinadas a alcanzar igualdad de género en las distintas áreas del quehacer universitario; prevenir y enfrentar la discriminación y violencia sexual, así como asesorar y acompañar a las personas afectadas; desarrollar políticas que permitan conciliar las responsabilidades familiares y el desempeño laboral y estudiantil; propiciar actividades académicas en docencia, investigación, creación y extensión e incidir en políticas públicas y normas de carácter nacional vinculadas con materias de género. Su organización y ubicación jerárquica constituyen una señal muy auspiciosa, ya que mejora su capacidad de incidencia y sitúa la equidad de género entre las prioridades institucionales. Asimismo, el apoyo del Consejo integrado por las autoridades responsables de las

6. "Proyecto de Ley Sobre el Derecho de las Mujeres a una Vida Libre de Violencia". Mensaje No307-364. Ingresado al Parlamento en noviembre, 2016.

7. Indicación presentada por los diputados Andrade, Saffirio y otros que plantea que "en el caso de las funcionarias del sector público deberá darse cumplimiento a las obligaciones establecidas en el presente artículo mediante lo dispuesto en el artículo primero o quinto, o mediante la provisión del servicio a través de los establecimientos de la Junta Nacional de Jardines Infantiles o de la Fundación Integra, en este último caso a elección de la funcionaria”. Boletín 9969-13, octubre 2016. 
funciones sustantivas y de la gestión universitaria facilita la transversalización de la perspectiva de género en todas las áreas como responsabilidad compartida por el conjunto de las unidades universitarias.

La institucionalidad de género se ha ido descentralizando paulatinamente en las diversas facultades e institutos; Ciencias Físicas y Matemáticas, Medicina, Odontología, Economía y Negocios, Asuntos Públicos, entre otras, han tomado la iniciativa de organizar unidades con distintos formatos: Dirección de Género y Diversidad, Comisión Triestamental, nombramiento de encargada de la temática y otros. Sin duda, conformar mecanismos de género en todas las unidades académicas sería un paso importante para desplegar las medidas y planes y a la vez para hacerlas más pertinentes a las realidades locales.

Por su parte, el Senado Universitario creó recientemente una Comisión de Género y en coordinación con la recién creada Dirección de Igualdad se incorporó en Plan de Desarrollo Institucional (PDI) ${ }^{\text {xvi }}$ que orientará la gestión de la Universidad por los próximos 10 años, un conjunto de objetivos estratégicos que constituirán una "carta de navegación” para avanzar en la institucionalización de la equidad de género en el desarrollo institucional orientados a garantizar:

I. igualdad de remuneraciones entre hombres y mujeres por funciones similares y/o de igual valor,

II. equidad en la composición de los comités académicos, espacios de representación, dirección y acceso a jerarquías,

III. igualdad de oportunidades de hombres y mujeres con responsabilidades familiares y de cuidado,

IV. la incorporación de la perspectiva de género en la docencia, generación de conocimiento, extensión universitaria y gestión institucional,

V. la erradicación del acoso sexual y la disminución de la violencia de género en el contexto universitario,

VI. el fortalecimiento de la Dirección de Igualdad de Género.

Otra iniciativa particularmente relevante que permitirá avanzar en el cumplimiento del compromiso de la Universidad de Chile con la educación no sexista fue iniciada por la Vicerrectoría de Asuntos Académicos, en coordinación con la DIGEN, con el propósito de incorporar la perspectiva de género en el modelo educativo de la Universidad y en distintos instrumentos tales como la encuesta de evaluación docente, la formación de nuevos académicos/as y de tutores, la revisión de prácticas educativas para identificar y erradicar sus rasgos sexistas, entre otros. "La Comisión de Modelo Educativo coordinada por la Vicerrectoría de Asuntos Académicos, a la que se integrará la Asamblea de Mujeres, será la encargada de 
proponer los principios de la educación no sexista que se aplicarán en los procesos formativos y de desarrollo académico"s.

Junto con impulsar la educación no sexista, las estudiantes de las asambleas de mujeres comprendieron claramente la importancia de "reforzar la institucionalidad de igualdad de género en la Universidad dadas las innegables desigualdades y discriminaciones que se aprecian en nuestra casa de estudios". Por este motivo, los acuerdos alcanzados producto de la movilización feminista en la Universidad destacan que:

- Se potenciará y reforzará la recientemente creada Dirección de Igualdad de Género. Su integración en la Rectoría permite que actúe coordinadamente con todo el equipo ejecutivo de la Universidad.

- La DIGEN reforzará la oficina de atención, defensa y acompañamiento de denunciantes y víctimas y contará) con un Consejo Asesor de carácter triestamental al que) se incorporará la representación de la Asamblea de Mujeres de la Universidad. Esta será la instancia encargada de proponer y evaluar políticas de equidad de género a los organismos universitarios correspondientes.

- El Consejo Consultivo tendrá carácter ejecutivo y será conformado por todas las vicerrectoras y vicerrectores, para transversalizar la perspectiva de igualdad de género en las funciones y la gestión universitaria.

- Se impulsarán dispositivos de igualdad de género a nivel local.

- Se creará una unidad central autónoma que ejercerá el rol de fiscalía y tendrá el propósito de mejorar el procedimiento de investigación de los casos de acoso, abuso sexual, violencia de género y discriminación arbitraria.

- Se creará también un Observatorio de Género que generará información sistemática y una Unidad de Salud Mental especializada para atender a personas afectadas por situaciones de violencia de género.

Previo a estos acuerdos y en plena sintonía con ellos, la Universidad, en el marco del Día Internacional de la Mujer en marzo de 2018, dio a conocer un estudio sobre brechas salariales en el estamento académico y funcionario y anunció que desarrollará un proceso de certificación en igualdad de género en coordinación con el Programa de la Naciones Unidas para el Desarrollo (PNUD). "Se propone diseñar e implementar un programa con la Universidad de Chile, que permita que

8. Acta de acuerdo entre la Rectoría de la Universidad de Chile y representantes de la Asamblea de Mujeres, junio de 2018.

9. Acta de Acuerdo, 2018. 
ésta sea la primera Universidad del país en realizar un proceso de certificación en estándares de igualdad de género y, al mismo tiempo, contribuya a reconocer los esfuerzos que hacen las instituciones académicas hacia el logro de la igualdad sustantiva entre hombres y mujeres en el marco de la Agenda 2030 de las Naciones Unidas" (PNUD, 2018).

Si bien los procesos de institucionalización de la equidad de género en el Estado y en la Universidad tienen evidentes especificidades, es posible destacar similitudes entre ambos. Por una parte, la influencia que ejerce la voluntad política de las autoridades; en el caso de la Universidad de Chile ha resultado gravitante la participación de la Rectoría, el Senado, las vicerrectorías y los decanatos, quienes junto con aprobar por unanimidad las nuevas iniciativas de género, las han ido vinculando con las definiciones generales, los valores y principios de la Universidad.

Por otra parte, la ejecución de políticas universitarias elaboradas con amplia participación de la comunidad, que se sustentan en marcos teóricos, discursos simbólicos y nuevas concepciones, han instalado nuevas conversaciones y han tensionado la institucionalidad mostrando formas concretas de enfrentar problemas de género y planteando a distintos actores institucionales la necesidad de revisar sus propias definiciones y acciones desde la perspectiva de género.

Asimismo, la generación de un mecanismo institucional especializado y el mejoramiento de su posición dentro de la estructura universitaria (desde una oficina dependiente de una vicerrectoría a una dirección dependiente de la Rectoría), así como la conformación paulatina de unidades locales, han contribuido a situar la equidad e igualdad de género como asunto prioritario y a iniciar la transversalización de esta perspectiva en las distintas áreas. Así también, las nuevas reglamentaciones y normativas, si bien han debido seguir un largo camino administrativo para su aprobación, ofrecen mayor sustento y estabilidad a las políticas de igualdad, a la vez que las proyectan más allá de un periodo de gobierno universitario.

En este sentido, la construcción de la institucionalidad de género en la Universidad es expresión tanto de relaciones y prácticas sociales como de visiones político-culturales e involucra normas, organismos institucionales, programas, servicios, organizaciones sociales e incluso áreas de conocimiento legitimadas.

La experiencia comparada de universidades extranjeras muestra que las políticas de género se instalan con más facilidad y aumentan su impacto cuando existen leyes nacionales que operan como "paraguas institucional" que las refuerzan y relacionan con políticas generales. En el país se carece de este instrumento y la Universidad de Chile, tomando en cuenta el potencial transformador de las instituciones de educación superior hacia la sociedad, podría jugar un rol importante en conjunto con las otras universidades públicas para instalar esta demanda en la agenda pública. 
Finalmente, uno de los aprendizajes importantes de las experiencias gubernamentales es que la agenda de género puede perder jerarquía y disiparse de las agendas institucionales. La idea de que esta "llegó para quedarse" no es automática sino que se sostiene de manera importante en la actuación permanente de actores universitarios que le dan fuerza y legitimidad. En este sentido, la construcción de alianzas, respetando las legítimas diferencias, entre los colectivos feministas, las asambleas de mujeres, los centros de estudios de género, las agrupaciones de académicas, la Dirección de Igualdad de Género, debieran favorecer la profundización de la equidad de género en la institución.

De este modo, se asume el compromiso de continuar desarrollando las prácticas históricas de los movimientos de mujeres y feministas que en distintos lugares, momentos y circunstancias, han cuestionado las injustas desigualdades entre hombres y mujeres, así como las lógicas y culturas de las instituciones que las reproducen, y han actuado unidas para transformarlas. 


\section{REFERENCIAS}

i Repetto, Fabián y Andrenacci, Luciano: Problemas de política social en la Argentina contemporánea (Pág.5). En "Ciudadanía y capacidad estatal: dilemas presentes en la reconstrucción de la política social argentina" (Repetto, Andrenacci compiladores). Edit. Prometeo, Buenos Aires, 2006.

ii Declaración de Santiago, Trigésima Cuarta Asamblea de Delegadas de la Comisión Interamericana de la Mujer (CIM), Organización de los Estados Americanos (OEA) Santiago, 2008.

iii Astelarra, Judith. Políticas de género en la Unión Europea y algunos apuntes sobre América Latina. Serie Mujer y Desarrollo No57. Comisión Económica para América Latina (CEPAL), Santiago 2004.

iv Economic and Social Council, ECOSOC. Gender mainstreaming. An overview. United Nations. New York, 2002.

$\mathrm{v}$ Los mecanismos de adelanto de las mujeres nacionales y regionales y sus posibilidades de incidencia en políticas públicas de género. Doc. Mesa Redonda Virtual de Especialistas (Pág.2). Instituto Internacional de Investigaciones y Capacitación para la Promoción de la Mujer (INSTRAW), Santo Domingo, 2010.

vi Guzmán, Virginia y Montaño, Sonia. Políticas públicas e institucionalidad de género 1985-2010. Serie Mujer y Desarrollo No118. Comisión Económica para América Latina (CEPAL), Santiago, 2012.

vii Benavente, María Cristina y Valdés, Alejandra. Políticas públicas para la igualdad de género. Un aporte a la autonomía de las mujeres (Pág.113). Comisión Económica para América Latina (CEPAL), Santiago, 2014.

viii Guzmán, Virginia. Sostenibilidad social y política de los mecanismos para el adelanto de las mujeres (Pag.2). Presentación realizada en Trigésima novena reunión de la Mesa Directiva de la Conferencia Regional sobre la Mujer de América Latina y el Caribe. Comisión Económica para América Latina (CEPAL). Ciudad de México, 2006.

ix Del biombo a la cátedra: igualdad de oportunidades de género en la Universidad de Chile. Investigación coordinada por Carmen Andrade Lara. Oficina de Igualdad de Oportunidades, Universidad de Chile, 2013.

x Buquet, Ana Gabriela. Transversalización de la perspectiva de género en la educación superior. Problemas conceptuales y prácticos. En Perfiles educativos | vol. XXXIII, número especial, UNAM, Ciudad de México 2011. 
xi Székely, Miguel. Midiendo el Nivel de institucionalidad de la política social en América Latina. Doc. Programa de Naciones Unidas para el Desarrollo (PNUD), Bogotá, 2006.

xii Universidad de Chile, 2013 Ob. cit.

xiii Cerva, Daniela. Desafíos para la institucionalización de la perspectiva de género en instituciones de educación superior en México. Una mirada a los contextos organizacionales (Pág.26). Revista Punto Género Nº8. Ciudad de México, 2017.

xiv Cerva, Daniela, Ob. cit. (Pág.30)

xv Guzmán, Virginia. Ob. cit.

xvi Plan de Desarrollo Institucional de la Universidad de Chile. Comisión de Desarrollo Institucional - Senado Universitario. Universidad de Chile, Santiago, 2018 (Cap. IX Pág.28). 